by a multidisciplinary team including doctors, nurses, educators and managers. Best available evidence, national guidance and local clinical agreement were used to design and implement the service.

3. In order to deliver the service a bespoke training package for nurses was developed which included the development of robust governance processes covering clinical and non-clinical interactions.

4. Four separate work-streams are led and managed to deliver the service: implementation, training and competence, pathways and clinical governance.

Results Dec 2017- June 2019

$83 \%$ of patients referred to the service have been managed entirely in the community. There has been $100 \%$ positive feedback and no adverse events.

Conclusion ACE is the result of system wide collaboration. The service provides specialist CYP and family centered acute care at home, consistent with the NHS Long Term Plan. It has been designed for adaptation and replication across the NHS. It is a good example of the healthcare leadership model and an innovative way of working, ensuring we can cater for the healthcare needs of our current and future population.

\section{DEVELOPING TOMORROWS LEADERS: TRAINEE LEADERSHIP ROLES}

Brendan Spooner, Chetan Parcha, Tomasz Torlinski. University Hospitals Birmingham, UK

\subsection{6/leader-2019-FMLM.94}

Background The GMC has set out leadership and management responsibilities for all doctors, ${ }^{1}$ however opportunities for trainees to gain exposure to these skills are not widespread, as post graduate training is predominantly clinical. An emerging way for trainees to gain these skills is by way of trainee leadership roles.

Discussion In an anaesthetic and critical care department there is opportunity for trainees to take on trainee leadership roles. There is a trainee leadership structure for senior trainees within the department mirroring many consultant roles (service lead/clinical director, governance, education, wellbeing, etc.). Candidates are selected by interview for 6-month posts. The programme began with a single trainee leader but has now expanded to 9 roles. The benefits to the department and ultimately patients have become more apparent as the structure has evolved, demonstrated by various improvements. Concerns raised by trainees via the trainee leads empowers them to design solutions. As the structure has evolved, the problem-solving capacity of the structure has improved such that problems are often presented to the consultant department leaders with sensible and practical solutions. Some issues like trainees missing training time are now entirely managed within the trainee leadership setup with regular reports to department leaders.

Furthermore, the benefits for the individual trainee are also significant for developing problem-solving, collaborative-working, communication and leadership skills.
Conclusion Engaged trainees can be enthusiastic and powerful agents for change when empowered and can improve patient care. Having a trainee leadership structure within a department allows trainees to develop into tomorrow's leaders.

\section{REFERENCES}

1. Leadership and management for all doctors. GMC, 2012. Available at: https:// www.gmc-uk.org/-/media/documents/leadership-and-management-for-all-doctorsenglish-1015_pdf-48903400.pdf(accessed 24/8/2019).

\section{COMPLEXITY SCIENCE IN CLINICAL ORTHOPAEDIC PRACTICE - IMPLICATIONS FOR ALL HEALTHCARE STAKEHOLDERS}

Sunny Deo*, Sergio Prada, Yassir Alarabi, Kareem Elsorafy. The Great Western Hospitals NHS Foundation Trust, Swindon, Wilts. UK

\subsection{6/leader-2019-FMLM.95}

Complexity principles, highlighting the importance of interaction of agents, system history, start point and the potential for emergent events, may provide a better medical model. Our observation was of escalating clinical complexity, ie the interaction between the severity of primary condition and general status. We sought to quantify this.

Patients, methodology and summary of results

Over the past 8 years we have undertaken a number of clinical studies centred on clinical complexity which creates 4 groups: straightforward C0, locally complex C1, systemically complex $\mathrm{C} 2$ and most complex C3, which takes the form of a $2 \times 2$ table, with escalating complexity from $\mathrm{C} 0$ to $\mathrm{C} 3$.

We have undertaken studies for both elective and trauma conditions. We have found statistically significant differences between straightforward and most complex patient groups in a variety of parameters such as complication and mortality rates, recovery times and cost.

The future We are currently analysing results of a much larger cohort of knee replacement patients, and are aiming to apply the methodology to hip replacement and tibial plateau fractures. We hope to roll this methodology out to other Trusts and feel the methodology is suitable for any index condition.

\section{Conclusions}

- Patients' clinical complexity is a key factor in determining treatment outcomes. This is currently not done adequately.

- It confirms summative interaction between local and general factors which is been under-represented in historic texts, literature and current registries.

- Complexity classification has increased the understanding of all the teams involved with patient care, improved patient counselling, surgical skillset and operating time allocation and cost awareness.

- The methodology fits with contemporary studies in other fields such as macro-economics.

- The methodology of application and use of this method of patient stratification is likely to have universal applicability. 